J. Bangladesh Acad. Sci., Vol. 42, No. 1, 67-71, 2018

\title{
CERTAIN PROPERTIES OF FUZZY PATH CONNECTEDNESS AND MAXIMAL FUZZY PATH CONNECTEDNESS
}

\author{
RUHUL AMIN*AND SOHEL RANA \\ Department of Mathematics, Faculty of Science, Begum Rokeya University, Rangpur-5404, \\ Bangladesh
}

\begin{abstract}
This paper deals with some properties of fuzzy path connectedness and maximal fuzzy path connectedness on a fuzzy topological space by means of fuzzy points.
\end{abstract}

Key words: Fuzzy point, Fuzzy path, Fuzzy path connectedness, Maximal fuzzy path connectedness.

\section{INTRODUCTION}

The concept of fuzzy sets was first introduced by L. A. Zadeh (1965). Later C. L. Chang (1968), R. Lowen (1976), Halakatti and Halijol (2016) and others developed the theory of fuzzy topological spaces. One intrinsic characteristic of fuzzy topological space is path connectedness. C. L. Chang (1968) first introduced fuzzy path and fuzzy path connectedness and D. M. Ali (1987) introduced the new concept of fuzzy path connected space and studied its various characteristics. The purpose of this paper is to further contribute to the development of fuzzy path connected spaces.

In the present paper, we introduced a new definition of fuzzy path, fuzzy path connectedness and maximal fuzzy path connectedness depending on fuzzy point. By using these definitions, we observe that fuzzy path connectedness and maximal fuzzy path connectedness forms an equivalence relation. Also, fuzzy path connected and maximal fuzzy path connected spaces are fuzzy connected. Further the image of a fuzzy path connected space and of a maximal fuzzy path connected space under fuzzy continuous mapping is fuzzy path connected and maximal fuzzy path connected, respectively.

\section{BASIC NOTIONS AND PRELIMINARY RESULTS}

In this section, we recall some concepts which will be needed in the sequel. In the present paper, $X$ and $Y$ always denote non-empty sets and $\mathrm{I}=\left[\begin{array}{ll}0 & 1\end{array}\right]$ is the closed unit interval.

Definition: Let $X$ be a set and $I^{X}$ denote the set of fuzzy sets in $X$. A fuzzy topology on $X$ is a subset $\delta \subset I^{X}$ such that

(i) $0,1 \in \delta$

(ii) $\forall \mu \in \delta$

(iii) $\forall\left(\mu_{i}\right)_{i \in \mathrm{I}} \subset \delta \Rightarrow \sup \mu_{i} \in \delta$

Then is $(X, t)$ called a fuzzy topological space or short fts. (Chang 1968).

\footnotetext{
* Corresponding author: <ruhulbru1611@gmail.com>.
} 
Definition: A fuzzy point $p$ in $X$ is a special type of fuzzy set with membership function

$$
p(x)=\left\{\begin{array}{l}
r \text { if } x=x_{0} \\
0 \text { if } x \neq x_{0}
\end{array}\right.
$$

where $0<r<1$. (Zadeh 1965)

Definition: Let $(X, t)$ and $(Y, s)$ be two fuzzy topological spaces. A function

$f:(X, t) \rightarrow(Y, s)$ is called fuzzy continuous iff $f^{-1} \in t$ for every $v \in s$. (Zadeh 1965)

Definition: A fuzzy topological space $X$ is said to be fuzzy connected iff $\mu \cup \lambda=1$, where $\mu$ and $\lambda$ are non-empty open fuzzy sets in $X$ such that $\mu \cap \lambda=0$. (SethupathyLakshmivarahan 1977)

Definition: Let $(X, t)$ be an fuzzy topological space and $I=\left[\begin{array}{ll}0 & 1\end{array}\right]$. A continuous mapping $f$ : $I \rightarrow X$ is said to be a fuzzy path in $X$ if for any two points $x, y \in X$ such that $f(0)=x$, $f(1)=y($ Ali 1987).

Definition: Let $f$ is a fuzzy path from $p$ to $q$ i.e., $f: I \rightarrow X$ such that $f(0)=p, f(1)=q$ and also let $g$ is a fuzzy path from $q$ to $r$ i.e., $g: I$ $\rightarrow X$ such that $g(0)=q, g(1)=r$.

Now define $h: I \rightarrow X$ by

$h(t)=\left\{\begin{array}{l}f(2 t) ; 0 \leq t \leq t \leq \frac{1}{2} \\ g(2 t) ; \frac{1}{2} \leq t \leq 1\end{array}\right.$

Since $f(1)=r=g(0), h$ is well defined and it is obviously a fuzzy continuous function. Then $h$ is a composite fuzzy path. (HalakattiHalijol 2016)

Definition: Considering a fuzzy path $f: I \rightarrow$ $X$ such that $f(x)=t, \forall x \in[0,1]$. Then $f$ is called a constant fuzzy path. (HalakattiHalijol 2016)

\section{MAIN RESULTS}

Definition: Let $(X, t)$ be a fuzzy topological space and $I=[0,1]$. A continuous mapping $f$ $: \mathrm{I} \rightarrow X$ is said to be a fuzzy path in $X$ if for any two fuzzy points $p, q \in X$ such that $f(0)$

$=p, f(1)=q$.

Definition: Any fuzzy topological space $(X, t)$ is said to be fuzzy path connected iff for every fuzzy point $p, q \in X$ there exists a fuzzy path $f$ in $X$ such that $f(0)=p$ and $f(1)=q$.

Theorem: Every fuzzy path connected space is fuzzy connected.

Proof: Let $X$ be fuzzy path connected and let $X=\mu \cup \lambda$, where $\mu$ and $\lambda$ are non-empty open fuzzy sets. We have to prove that $\mu \cap \lambda \neq \phi$.

Suppose $\mu \cap \lambda \neq \phi$. Taking two fuzzy points $p$ $\in \mu$ and $q \in \lambda$. Then $p, q \in X$. As $X$ is fuzzy path connected, then there exists a fuzzy path $f$ : $\mathrm{I} \rightarrow X$ such that $f(0)=p, f(1)=q$.

As $p \in \mu, q \in \lambda$ and $f$ is a fuzzy path from fuzzy point $p$ to the fuzzy point $q$ which gives a contradiction. Hence $\mu \cap \lambda \neq \phi$. Therefore $X$ is fuzzy connected.

Theorem: Let $X$ and $Y$ be fts and $f: \mathrm{X} \rightarrow Y$ be an onto fuzzy continuous function. If $X$ is fuzzy path connected then $Y$ is fuzzy path connected.

Proof: Let $X$ is fuzzy path connected and $p, q$ be the two fuzzy points in $Y$. Then by definition of fuzzy continuous function, we have $f^{-1}(p)$ and $f^{-1}(q)$ is in $X$. Since $X$ is fuzzy path connected, then there exists a fuzzy path $g$ (say) from $f^{-1}(p)$ to $f^{-1}(q)$ is in $X$, i.e., $g: I$ $\rightarrow X$ is a fuzzy path such that $\mathrm{g}(0)=f^{-1}(p)$ and $f^{-1}(q)$. 
Now consider the function $f g: \rightarrow Y$. Then $(f g)(0)=f(g(0))=f^{-1}(q)=p$ and $(f g)(1)=$ $f(g(1))=f\left(f^{-1}(q)\right)=p$.

Thus $f g: I \rightarrow Y$ is a fuzzy path in $Y$ from $p$ to $q$. Therefore, $Y$ is fuzzy path connected.

Theorem: Let $\left\{X_{i}\right\}$ be a collection of fuzzy path connected spaces and $\cap X_{i}=\phi$. Then $X$ $=\cup X_{i}$. Then $X=\cup X_{i}$ is fuzzy path connected.

Proof: Let $p, q$ be the two fuzzy points in $X$, then for some $i_{1}$ and $i_{2}$, we have $p \in X_{i_{1}}, q \in X_{i_{2}}$.

Let $r$ be the fuzzy point in $X_{i_{1}} \cap X_{i_{2}}$. Since $X_{i_{1}}$ is fuzzy path connected, then there exists a fuzzy path $f: I \rightarrow X_{i_{1}}$ from $p$ to $r$ such that $f(0)=p$ and $f(1)=r$.

Similarly, for $X_{i_{2}}$, we get a fuzzy path $g: I \rightarrow X_{i_{2}}$ from $r$ to $q$ such that $g(0)=r$, $g(1)=q$.

Now define $h(t)=\left\{\begin{array}{l}f(2 t) ; 0 \leq t \leq \frac{1}{2} \\ g(2 t) ; \frac{1}{2} \leq t \leq 1\end{array}\right.$

Since $f(1)=r=g(0), h$ is well defined and $h$ is fuzzy continuous mapping.

Now $h(0)=f(0)=p$ and $h(1)=g(1)=q$. Thus $h$ is a fuzzy path in $X$. Therefore $X$ is fuzzy path connected.

Theorem: Fuzzy path connectedness of fuzzy topological spaces is an equivalence relation.

Proof: Let $X$ be a fuzzy path connected space.

Reflexivity : Reflexive relation is trivial by considering a constant fuzzy path $f: I \rightarrow X$ such that $f(t)=p \forall t \in\left[\begin{array}{ll}0 & 1\end{array}\right]$.
Symmetry : Suppose $f$ is a fuzzy path from the fuzzy $p$ to the fuzzy point $q$ such that $f(0)$ $=p, f(1)=q$.

Now define $g: I \rightarrow X$ such that $g(t)=f(1-\mathrm{t})$ $\forall t \in\left[\begin{array}{ll}0 & 1\end{array}\right]$.

Now $g(0)=f(1)=q$ and $g(1)=f(1)=p$ and $g$ is fuzzy continuous mapping.

Hence $g: I \rightarrow X$ is a fuzzy path from $q$ to $p$. Therefore, fuzzy path connected relation is symmetric relation.

Transitivity : Consider $p, q$ and $r$ be the fuzzy points in $X$. Suppose $f: I \rightarrow X$ is a fuzzy path such that $f(0)=p, f(1)=r$ and $g: I \rightarrow X$ is a fuzzy path such that $g(0)=r, g(1)=q$.

Now define $h(t)=\left\{\begin{array}{l}f(2 t) ; 0 \leq t \leq \frac{1}{2} \\ g(2 t) ; \frac{1}{2} \leq t \leq 1\end{array}\right.$

As $f(1)=r=g(0)$ then $h$ is well defined and $h$ is fuzzy continuous. Now $h(0)=f(0)=p$ and $h(1)=f(1)=q$. Thus $h$ is a fuzzy path from $p$ to $q$. Hence fuzzy path connected relation is transitive relation.

Therefore, fuzzy path connectedness is an equivalence relation.

Definition: Let $X$ be a fuzzy topological space and $p, q, r$ be the fuzzy points in $X$. If there exists fuzzy continuous mapping $f: I$ $\rightarrow X$ such that

$f(0)=p, f\left(\frac{1}{2}\right)=q, f(1)=r$

then $p$ is a maximal fuzzy path connected to $r$. If it is true for all $p, q, r \in X$, then $X$ is a maximal fuzzy path connected space.

Theorem: Every maximal fuzzy path connected space is fuzzy connected. 
Proof: Let $X$ be a maximal fuzzy path connected space. Le $X=\mu \cup \lambda$, where $\mu$ and $\lambda$ are non-empty open fuzzy sets in $X$. Now we have to show that $\mu \cap \lambda \neq \phi$.

Suppose $\mu \cap \lambda \neq \phi$. Suppose that $p, q$ be two fuzzy points in $\mu$ and $r$ be the fuzzy point in $\lambda$. This implies that $p, q, r \in X$. Since $X$ is maximal fuzzy path connected, so that there exists a maximal fuzzy path $f: I \rightarrow X$ such that

$$
f(0)=p, f\left(\frac{1}{2}\right)=q, f(1)=r .
$$

This is a contradiction.

Similarly, the same contradiction arise if we let $p$ be the fuzzy point in $\mu$ and $q, r$ be the fuzzy points in $\lambda$. Hence $\mu \cap \lambda \neq \phi$. Thus $X$ is fuzzy connected space.

Theorem: Let $X$ and $Y$ be fuzzy topological spaces and $f: X \rightarrow Y$ be an onto fuzzy continuous function. If $X$ is maximal fuzzy path connected space then $Y$ is so.

Proof: Let $X$ be maximal fuzzy path connected and $p, q, r$ be the fuzzy points in $Y$. Then by definition of fuzzy continuous function, we have $f^{-1}(p), f^{-1}(q), f^{-1}(r)$ in $X$. Since $X$ is maximal fuzzy path connected space, then there exists a maximal fuzzy path $g: I \rightarrow X$ such that

$g(0)=f^{-1}(p), g\left(\frac{1}{2}\right)=f^{-1}(q), g(1)=f^{-1}(r)$.

Consider the function fog $: I \rightarrow Y$ such that

$(f \circ g)(0)=f(g(o))=f\left(f^{-1}(p)\right)=p$

$($ fog $)\left(\frac{1}{2}\right)=f\left(g\left(\frac{1}{2}\right)\right)=f\left(f^{-1}(q)\right)=q$

$(f \circ g)(1)=f(g(1))=f\left(f^{-1}(r)\right)=r$.
This implies that fog is a maximal fuzzy path in $Y$ from $p$ to $r$. Therefore, $Y$ is maximal fuzzy path connected space.

Theorem: Maximal fuzzy path connectedness of a fuzzy topological space is an equivalence relation.

Proof: Let $X$ be a fuzzy topological space. Now we shall show that maximal fuzzy path connectedness is an equivalence relation.

Reflexivity : Reflexive relation is trivial by considering a constant fuzzy path $f: I \rightarrow X$ such that $f(p)=t, \forall p \in\left[\begin{array}{ll}0 & 1\end{array}\right]$. Therefore, maximal fuzzy path connectedness is a reflexive relation.

Symmetry : Suppose $f$ is a maximal fuzzy path from the fuzzy point $p$ to the fuzzy point $r$ i.e., path $f: I \rightarrow X$ such that

$$
f(0)=p, f\left(\frac{1}{2}\right)=q, f(1)=r .
$$

Now we define $g: I \rightarrow X$ such that $g(t)=f(1-t), \forall t \in\left[\begin{array}{ll}0 & 1\end{array}\right]$. It is clear that $g$ is fuzzy continuous and hence a maximal fuzzy path in $X$ from $r$ to $p$ such that

$g(0)=f(1-0)=f(1)=r$,

$g\left(\frac{1}{2}\right)=f\left(1-\frac{1}{2}\right)=f\left(\frac{1}{2}\right)=q$

$g(1)=f(1-1)=f(0)=p$.

Therefore, maximal fuzzy path connectedness is a symmetric relation.

Transitivity : Let $f$ is a maximal fuzzy path from the fuzzy point $p$ to the fuzzy point $r$ i.e., $f: I \rightarrow X$ such that

$f(0)=p, f\left(\frac{1}{2}\right)=q, f(1)=r$ and also let $g$ is a maximal fuzzy path from the fuzzy point $r$ to the fuzzy point $t$ i.e., $g: I \rightarrow X$ such that 


$$
g(0)=r, g\left(\frac{1}{2}\right)=s, g(1)=t .
$$

Now define $h: I \rightarrow X$ such that $h(t)$

$$
=\left\{\begin{array}{l}
f(2 t) ; 0 \leq t \leq \frac{1}{2} \\
g(2 t) ; \frac{1}{2} \leq t \leq 1
\end{array}\right.
$$

Since $\mathrm{f}(1)=\mathrm{r}=\mathrm{g}(0)$, then $h$ is well defined and it is obviously a fuzzy continuous function.

Now $h(0)=f(0)=p$

$h\left(\frac{1}{2}\right)=\left\{\begin{array}{l}f\left(2 \times \frac{1}{2}\right)=f(1)=r ; 0 \leq t \leq \frac{1}{2} \\ g\left(2 \times \frac{1}{2}-1\right)=g(0)=r ; \frac{1}{2} \leq t \leq 1\end{array}\right.$

$$
h(1)=g(1)=t .
$$

Therefore, $h$ is a maximal fuzzy path from $p$ to $t$. Thus, maximal fuzzy path connectedness is transitive relation. Therefore, maximal fuzzy path connectedness is an equivalence relation.

\section{REFERENCES}

Ali, D. M. and Arun K. Srivastava. 1988. On fuzzy connectedness. Fuzzy Sets and Systems. 28: 203-208.

Ali, D. M. 1987. On fuzzy path connectedness. BUSEFAL. 26-33.
Ali, D. M. 1992. Some other types of fuzzy connectedness. Fuzzy Sets and Systems. 46: 56-61.

Ajmal, N. and Kohili. 1989. Connectedness in fuzzy topological spaces. Fuzzy sets and systems. 31: 369-388.

Chang, C. L. 1968. Fuzzy topological spaces. J. Math. Annal. Appl. 24: 182-190.

Halakatti, S. C. P. and A. Halijol. 2016. Different types of fuzzy path connectedness on fuzzy Banach Manifold. IOSR Journal of Mathematics (IOSR-JM). 12(3): 74-78.

Lowen, R. 1976. Fuzzy topological space and compactness. J. Math. Annal. Appl. 56: 621633.

Ming, P. P., and L. Y. Ming. 1980. Fuzzy topology I. Neighbourhood structure of a fuzzy point and Moore-Smith convergence. J. Math. Annal. Appl. 76: 571-599.

Ming, P. P., and L. Y. Ming. 1980. Fuzzy topology II. Product and quotient spaces. $J$. Math. Annal. Appl. 77: 20-37.

Sethupathy, K. S. R., and S. Lakshmivarahan. 1977. Connectedness in fuzzy topology. KYBERNETIKA. 13(3).

Sohel Rana, R. Amin, S. S. Miah and R. Islam. 2017. Studies on fuzzy connectedness. $J$. Bangladesh Academy of Sciences. 41(2): 175182.

Wuyts, P. 1987. Fuzzy path and fuzzy connectedness. Fuzzy sets and Systems. 24: 127-128.

Zadeh, L. A. 1965. Fuzzy sets. Information and control. 8: 338-353.

Zheng, C. Y. 1984. Fuzzy path and fuzzy connectedness. Fuzzy Sets and Systems. 14: 273-280.

(Received revised manuscript on 17 April, 2018) 